\title{
The Same Core Rhythm Generator Underlies Different Rhythmic Motor Patterns
}

\author{
Rachel S. White and Michael P. Nusbaum \\ Department of Neuroscience, Perelman School of Medicine, University of Pennsylvania, Philadelphia, Pennsylvania 19104-6074
}

\begin{abstract}
Rhythmically active motor circuits can generate different activity patterns in response to different inputs. In most systems, however, it is not known whether the same neurons generate the underlying rhythm for each different pattern. Thus far, information regarding the degree of conservation of rhythm generator neurons is limited to a few pacemaker-driven circuits, in most of which the core rhythm generator is unchanged across different output patterns. We are addressing this issue in the network-driven, gastric mill (chewing) circuit in the crab stomatogastric nervous system. We first establish that distinct gastric mill motor patterns are triggered by separate stimulation of two extrinsic input pathways, the ventral cardiac neurons (VCNs) and postoesophageal commissure (POC) neurons. A prominent feature that distinguishes these gastric mill motor patterns is the LG (lateral gastric) protractor motor neuron activity pattern, which is tonic during the VCN rhythm and exhibits fast rhythmic bursting during the POC rhythm. These two motor patterns also differed in their cycle period and some motor neuron phase relationships, duty cycles, and burst durations. Despite the POC and VCN motor patterns being distinct, rhythm generation during each motor pattern required the activity of the same two, reciprocally inhibitory gastric mill neurons [LG, Int1 (interneuron 1)]. Specifically, reversibly hyperpolarizing LG or Int1, but no other gastric mill neuron, delayed the start of the next gastric mill cycle until after the imposed hyperpolarization. Thus, the same circuit neurons can comprise the core rhythm generator during different versions of a network-driven rhythmic motor pattern.
\end{abstract}

\section{Introduction}

Rhythm generation is a key feature of many neuronal networks, including central pattern generators (CPGs) (Marder and Calabrese, 1996; Huguenard and McCormick, 2007; Mann and Paulsen, 2007; Welsh et al., 2010). Individual CPGs can generate different activity patterns when influenced by distinct inputs (Marder et al., 2005; Briggman and Kristan, 2008; Doi and Ramirez, 2008; Rauscent et al., 2009; Klein et al., 2010). The different motor patterns generated by a CPG involve changes in the relative timing, firing rate, burst duration, and/or burst pattern of one, some, or all of the associated motor neurons (Marder and Calabrese, 1996; Marder and Bucher, 2001; Buschges et al., 2008; Friedman et al., 2009; Rosenbaum et al., 2010). These different patterns underlie different variants of a behavior (e.g., different chewing patterns) or distinct behaviors (e.g., ingestion vs egestion).

The different patterns generated by a network often result, at least partly, from activating overlapping but distinct sets of network neurons (Jing and Weiss, 2002; Popescu and Frost, 2002; Proekt et al., 2007; Briggman and Kristan, 2008; Berkowitz et al.,

Received April 14, 2011; revised June 13, 2011; accepted June 15, 2011.

Author contributions: R.S.W. and M.P.N. designed research; R.S.W. performed research; R.S.W. analyzed data; R.S.W. and M.P.N. wrote the paper.

This work was supported by National Institutes of Neurological Disorders and Stroke Grants R37-NS 29436 and R01-NS 42813 (M.P.N.). We thank Dawn M. Blitz for helpful discussions and comments on previous versions of this manuscript and Dirk Bucher and Joshua Gold for assistance with data analysis.

Correspondence should be addressed to Dr. Michael P. Nusbaum, Department of Neuroscience, 215 Stemmler Hall, Perelman School of Medicine, University of Pennsylvania, Philadelphia, PA 19104-6074. E-mail: nusbaum@mail.med.upenn.edu.

DOI:10.1523/JNEUROSCI.1885-11.2011

Copyright $\odot 2011$ the authors $\quad 0270-6474 / 11 / 3111484-11 \$ 15.00 / 0$
2010; Weaver et al., 2010), although the same network neurons can also generate distinct patterns (Marder and Bucher, 2007). For some CPGs, some or all of the core rhythm-generating neurons for a particular motor pattern are identified (Selverston and Miller, 1980; Hooper and Marder, 1987; Masino and Calabrese, 2002; Cangiano and Grillner, 2003, 2005; Saideman et al., 2007; Li et al., 2010). Less is known regarding whether the different motor patterns generated by any single network are driven by the same rhythm-generating neurons. Thus far, this latter issue has been addressed primarily in CPGs driven by endogenously oscillatory neurons (pacemaker-driven CPGs). Work from these circuits provides contradictory examples, including preservation of the rhythm generator neurons across distinct motor patterns (Marder and Bucher, 2007; Weaver et al., 2010) and an apparent switch in the rhythm generator neurons (Peña et al., 2004). The flexibility of rhythm generator neurons in CPGs with no endogenously oscillatory neurons (network-driven CPGs) remains unexplored.

Here we assess the degree of preservation of the rhythm generator underlying different motor patterns generated by the networkdriven gastric mill (chewing) circuit in the isolated crab stomatogastric nervous system (STNS). Qualitatively distinct gastric mill patterns are triggered by the ventral cardiac neurons (VCNs) and postoesophageal commissure (POC) neurons (Beenhakker et al., 2004; Blitz et al., 2008).

We first establish that the VCN- and POC-gastric mill motor patterns are quantitatively distinct in their cycle period, phase durations, protractor LG (lateral gastric) neuron burst pattern, and the burst parameters of several other motor neurons. We then show that these differences result partly from the 


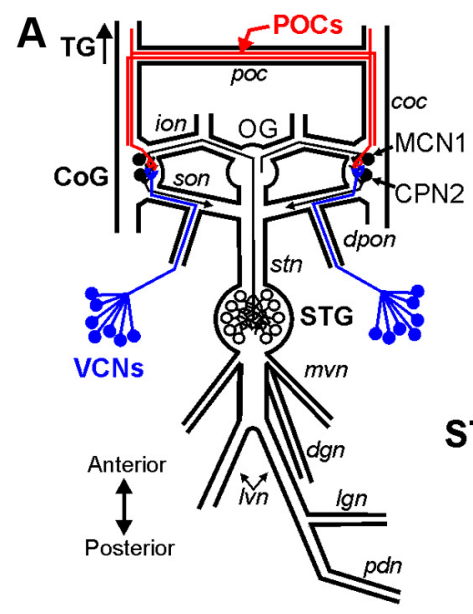

C Control Post-VCN stim.

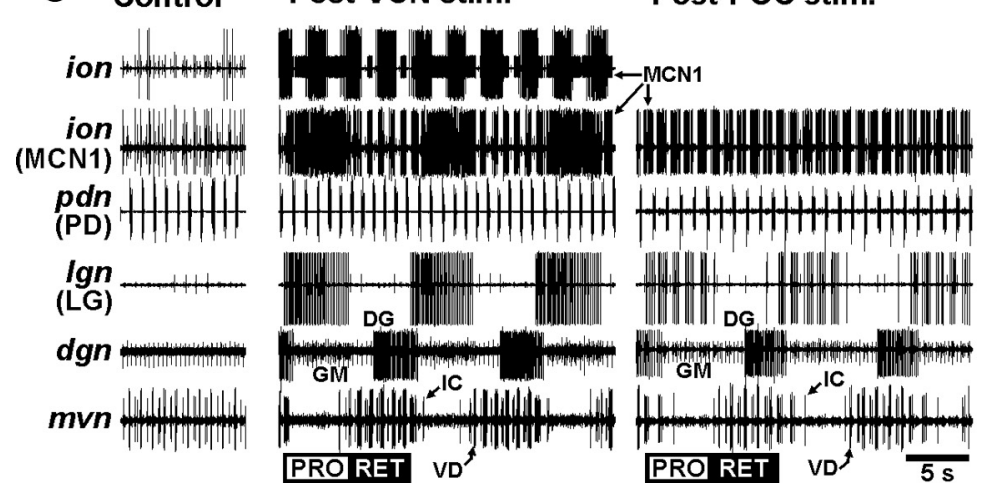

Figure 1. The VCN and POC pathways each trigger a gastric mill motor pattern. $A$, Schematic of the isolated STNS, including its four ganglia plus their connecting and peripheral nerves. The VCNs project into the CoGs from the cardiac sac stomach compartment via the dpon and son nerves. The POC neurons project into the CoGs via the coc and poc nerves. The single MCN1 and CPN2 projection neurons in each $\mathrm{COG}$ extend their axons to the STG via the ion and son, respectively, and then converge to reach the STG via the stn nerve. Ganglia: $\mathrm{COG}$, commissural ganglion; $0 \mathrm{G}$, oesophageal ganglion; STG, stomatogastric ganglion; TG, thoracic ganglion. Nerves: coc, circumoesophageal commissure; dgn, dorsal gastric nerve; dpon, dorsal posterior oesophageal nerve; ion, inferior oesophageal nerve; Ign, lateral gastric nerve; Ivn, lateral ventricular nerve; $m v n$, medial ventricular nerve; $p d n$, pyloric dilator nerve; poc, postoesophageal commissure; son, superior oesophageal nerve; stn, stomatogastric nerve. Neurons: CPN2, commissural projection neuron 2; MCN1, modulatory commissural neuron 1; POCs, postoesophageal commissure neurons; VCNs, ventral cardiac neurons. $\boldsymbol{B}$, Schematic of the gastric mill circuit activated by the VCN and POC pathways. As indicated, the top row of gastric mill neurons in the STG represent protractor (PRO) phase neurons, whereas the second row represent retractor (RET) phase neurons. Bottom row shows the pyloric pacemaker neurons. The exact electrical coupling relationship among the protractor neurons is not known, so they are shown simply as being serially coupled. All STG circuit neurons occur as single copies per STG, except for GM (4) and PD (2). Downward arrows, Activation of the system within the target box; upward arrow, synaptic feedback; filled circles, fast synaptic inhibition; resistor, nonrectifying electrical coupling; diode, rectifying electrical coupling. C, Gastric mill motor patterns triggered by brief stimulation of the VCN and POC pathways and recorded extracellularly from nerve branches shown schematically in $A$. No gastric mill rhythm was in progress before either pathway was stimulated, but the pyloric rhythm was ongoing (pdn, mvn: Control) and there was modest MCN1 activity (ion). In the Control and Post-VCN stimulation panels, which came from the same experiment, the lower ion recording is the same as the upper ion recording except that the large unit (an oesophageal rhythm motor neuron) was digitally subtracted to more explicitly show the MCN1 activity pattern (Blitz and Nusbaum, 2008). Also, the gain was increased in the lower ion recording to increase the amplitude of the MCN1 spikes. The large motor neuron unit in the ion was not active during the POC rhythm. Note that the protraction phase burst pattern of MCN1 and LG (Ign) was tonic during the VCN-gastric mill rhythm but was pyloric rhythm timed (see pdn) during the POC-gastric mill rhythm. The CPN2 burst pattern during each rhythm is the same as the MCN1 pattern (data not shown) (Beenhakker and Nusbaum, 2004; Blitz and Nusbaum, 2008). The POC- and VCN-gastric mill rhythms were recorded in separate preparations.

distinct influence of the pyloric rhythm on these two gastric mill rhythms. Last, we determine that, among the eight types of gastric mill neurons, only the reciprocally inhibitory LG and Int1 (interneuron 1) are necessary for gastric mill rhythm generation after VCN or POC stimulation. These results illustrate that the same core rhythm generator can pace different motor patterns generated by a network-driven motor circuit.

\section{Materials and Methods}

Animals. Male Jonah crabs (Cancer borealis) were obtained from commercial suppliers (Yankee Lobster; Marine Biological Laboratory) and maintained in aerated, filtered artificial seawater at $10-12^{\circ} \mathrm{C}$. Animals were cold anesthetized by packing in ice for at least $30 \mathrm{~min}$ before dissection. The foregut was then removed from the animal, after which the STNS was dissected from the foregut in physiological saline at $\sim 4^{\circ} \mathrm{C}$.

Solutions. C. borealis physiological saline contained the following (in $\mathrm{mm}$ ): $440 \mathrm{NaCl}, 26$ $\mathrm{MgCl}_{2}, 13 \mathrm{CaCl}_{2}, 11 \mathrm{KCl}, 10$ Trisma base, 5 maleic acid, and 5 glucose, $\mathrm{pH}$ 7.4-7.6. All preparations were superfused continuously with $C$. borealis saline $\left(8-12^{\circ} \mathrm{C}\right)$.

Electrophysiology. Electrophysiology experiments were performed using standard techniques for this system (Beenhakker and Nusbaum, 2004). The isolated STNS (Fig. 1A) was pinned down in a silicone elastomer-lined (Sylgard 184; KR Anderson) Petri dish. Each extracellular nerve recording was made using a pair of stainless steel wire electrodes (reference and recording) whose ends were pressed into the Sylgard-coated dish. A differential AC amplifier (model 1700; A-M Systems) amplified the voltage difference between the reference wire, placed in the bath, and the recording wire, placed near an individual nerve and isolated from the bath by petroleum jelly (Vaseline; Lab Safety Supply). This signal was then further amplified and filtered (model 410 amplifier; Brownlee Precision). Extracellular nerve stimulation was accomplished by placing the pair of wires used to record nerve activity into a stimulus isolation unit (SIU 5; Astro-Med/Grass Instruments) that was connected to a stimulator (model S88; Astro-Med/Grass Instruments).

Stimulation of the POC neurons was performed via extracellular stimulation of the postoesophageal commissure ( $p o c$ ) (Fig. 1), using a tonic stimulation pattern (duration, 15-30 s; intraburst frequency, $15-30 \mathrm{~Hz}$ ) (Blitz et al., 2008). In all experiments, the poc was bisected, and each half was surrounded by a petroleum jelly well to stimulate them separately. However, the left and right pocs were stimulated simultaneously in all experiments. The VCNs were activated by stimulating one or both of the dorsal posterior oesophageal nerves (dpons) (Fig. 1) in a rhythmic pattern (interburst frequency, $0.06 \mathrm{~Hz}$; burst duration, $6 \mathrm{~s}$; intraburst frequency, $15 \mathrm{~Hz}$ ) (Beenhakker et al., 2004). However, the same gastric mill motor pattern (e.g., cycle period, LG duty cycle) is triggered when the VCNs are stimulated in (1) the aforementioned rhythmic pattern, (2) a faster, pyloric rhythm-like pattern, or (3) a tonic pattern (Beenhakker et al., 2004).

Intrasomatic recordings were made with sharp glass microelectrodes (15-30 M $\Omega$ ) filled with $0.6 \mathrm{M} \mathrm{K}_{2} \mathrm{SO}_{4}$ plus 10 $\mathrm{mm} \mathrm{KCl}$. Intracellular signals were amplified using Axoclamp 2B amplifiers (Molecular Devices) and then further amplified and filtered (Brownlee model 410 amplifier). Current injections were performed in single-electrode discontinuous current-clamp mode with sampling rates between 2 and $3 \mathrm{kHz}$. To facilitate intracellular recording, the desheathed ganglia were viewed with light transmitted through a dark-field con- 
denser (Nikon). Stomatogastric ganglion (STG) neurons were identified on the basis of their axonal projections, activity patterns, and interactions with other STG neurons (Weimann et al., 1991; Blitz et al., 2008).

Data analysis. Data were collected in parallel onto a chart recorder (AstroMed Everest) and computer. Acquisition onto computer (sampling rate, $\sim 5$ $\mathrm{kHz}$ ) used the Spike2 data acquisition and analysis system (Cambridge Electronic Design). Some analyses, including cycle period, burst durations, duty cycle, number of action potentials per burst, interspike interval durations, intraburst firing frequency and phase relationships were conducted on the digitized data using a custom-written Spike2 program (The Crab Analyzer: freely available at http://www.uni-ulm.de/ wstein/spike2/index.html).

Unless otherwise stated, each data point in a dataset was derived by determining the mean for the analyzed parameter from 10 consecutive gastric mill cycles. One gastric mill cycle was defined as extending from the onset of consecutive LG neuron action potential bursts (Beenhakker and Nusbaum, 2004; Wood et al., 2004). Thus, the gastric mill cycle period was measured as the duration (seconds) between the onset of two successive LG neuron bursts. The protractor phase was measured as the LG burst duration, whereas the retractor phase was measured as the LG interburst duration. A gastric mill rhythmtimed burst duration was defined as the duration (seconds) between the onset of the first and last action potential within an impulse burst, during which no interspike interval was longer than $2 \mathrm{~s}$ (approximately twice the pyloric cycle period during the gastric mill rhythm and no more than half the duration of each gastric mill phase) (Beenhakker et al., 2004). The intraburst firing rate of a neuron was defined as the number of action potentials minus one, divided by the burst duration. The instantaneous spike frequency was defined as the inverse of each successive interspike interval within a burst. Duty cycle, defined as the fraction of a gastric mill cycle during which a particular neuron fired its burst, was determined by dividing each burst duration by the cycle period during which that burst occurred. The burst relationship among gastric mill neurons was expressed in terms of phase. Phase relationships were determined by measuring the phase of burst onset and offset for each gastric mill neuron relative to the gastric mill cycle. The onset and offset phase of each gastric mill neuron was determined as the latency from cycle onset to the start and endpoint, respectively, of a gastric mill neuron burst, divided by the cycle period.

We determined the LG burst pattern during the POC- and VCN-gastric mill rhythms with respect to the pyloric rhythm by determining its relationship to the activity of the pyloric dilator (PD) neuron, a member of the pyloric pacemaker ensemble, during normalized pyloric cycles. The normalized pyloric cycle extended from PD neuron burst onset to the start of the next PD burst, as is standard for the pyloric rhythm (Bucher et al., 2006). Specifically, we separated the LG recording during each normalized pyloric cycle into 100 equal bins ( 1 bin $=1 \%$ normalized pyloric cycle) and determined the fraction of the LG spikes during each pyloric cycle that occurred in each bin (Bucher et al., 2006). In general, there are several pyloric cycles per LG burst, insofar as the LG burst is $\sim 5 \mathrm{~s}$ duration, and the pyloric cycle period during these gastric mill rhythms is $\sim 1 \mathrm{~s}$ (Beenhakker and Nusbaum, 2004; Beenhakker et al., 2004; Blitz et al., 2008).

To determine whether each type of gastric mill neuron was necessary for gastric mill rhythm generation, activity in a gastric mill neuron was reversibly suppressed by hyperpolarizing current (range, -0.5 to -4.0 $\mathrm{nA}$ ) during an ongoing VCN- or POC-gastric mill rhythm. These hyperpolarizing current injections typically hyperpolarized the injected neuron to -65 to $-90 \mathrm{mV}$. This range of current injections was not sufficient to alter the activity of any neurons to which the hyperpolarized neuron is electrically coupled (see circuit diagram in Fig. $1 A$ ). In C. borealis, all gastric mill neurons except for the gastric mill (GM) motor neurons are present as single copies in each STG (Kilman and Marder, 1996). There
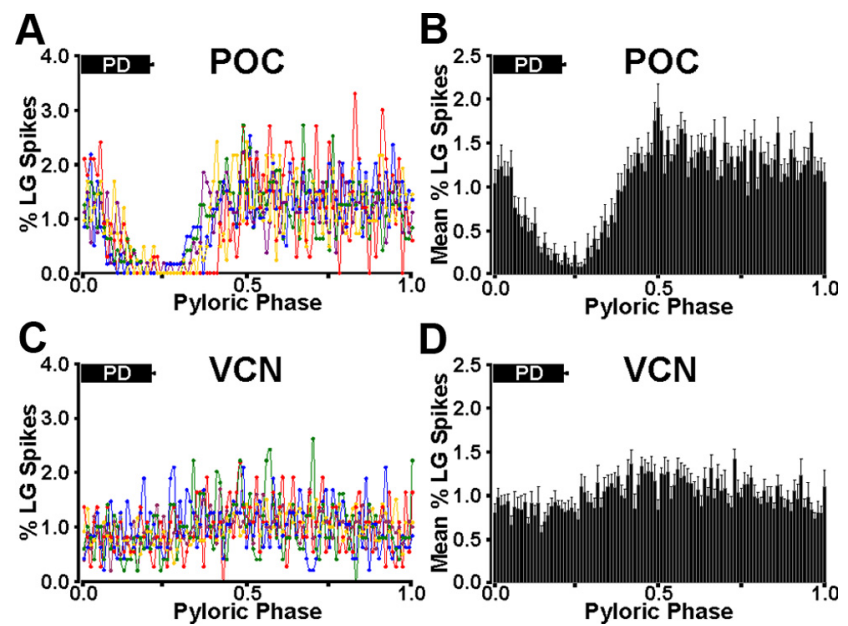

Figure 2. Quantitative analysis of the $L G$ burst structure during the POC- and VCN-gastric mill rhythms relative to $P D$ neuron activity. $A$, Mean percentage of $L G$ spikes per bin across the normalized pyloric cycle during the $\mathrm{POC}$-gastric mill rhythm, plotted separately for five different preparations, $10 \mathrm{LG}$ bursts/preparation (see Materials and Methods). The mean fraction of the normalized cycle during which PD was active is shown by the PD bar at the top of the graph. Note the consistent drop in LG activity during and immediately after the PD burst. Each color represents a single experiment. $\boldsymbol{B}$, Mean \pm SE percentage of the total $L G$ spikes across the normalized pyloric cycle for POC-gastric mill rhythms from 10 separate preparations, including the five experiments shown in A. C, Mean percentage of $L G$ spikes per bin across the normalized pyloric cycle during the VCN-gastric mill rhythm, plotted separately for five different preparations, 10 LG bursts/preparation. Note the consistent absence of a drop in LG activity during and after the PD burst. D, Mean \pm SE percentage of $L G$ spikes per bin across the normalized pyloric cycle from 10 separate VCN-gastric mill rhythms, including the five experiments shown in $\mathbf{C}$.
A

Figure 3. Comparison of $\mathrm{POC}$ - and VCN-gastric mill rhythm parameters. $A$, The POC-gastric mill cycle period and retracwhereas the DG neuron burst offset is phase advanced during the POC rhythm. Black bars, POC rhythm $(n=10)$; gray bars, VCN rhythm $(n=10) ;{ }^{*} p<0.05$.

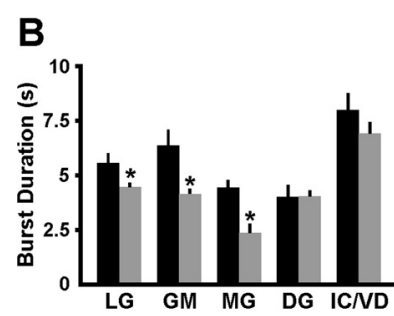

are four GM neurons per STG in C. borealis. Hence, for these experiments, three of four GM neurons were recorded intracellularly, and hyperpolarizing current was injected simultaneously into each one.

We determined whether a particular hyperpolarizing current injection altered the ongoing gastric mill rhythm by tracking the gastric mill cycle period via the rhythmic bursting in the LG neuron, except during LG hyperpolarizations when we also tracked Intl activity. To this end, we determined the mean gastric mill cycle period (successive LG burst onsets) for the five cycles before a hyperpolarizing current injection and then labeled the expected onset time of the next several gastric mill cycles in the absence of any perturbation of the ongoing rhythm. We then determined whether the first gastric mill cycle onset (LG burst onset) after the start of a hyperpolarizing current injection occurred at the expected time in the absence of the hyperpolarization.

Data were plotted with Excel (version 2002; Microsoft), Prism (version 3.0; GraphPad Software), and Matlab (MathWorks). Figures were produced using CorelDraw (version 13.0 for Windows). Statistical analyses were performed with Microsoft Excel, SigmaStat 3.0 (SPSS Inc.), and Matlab. Comparisons were made to determine statistical significance 


\section{A POC-Gastric Mill Rhythm}

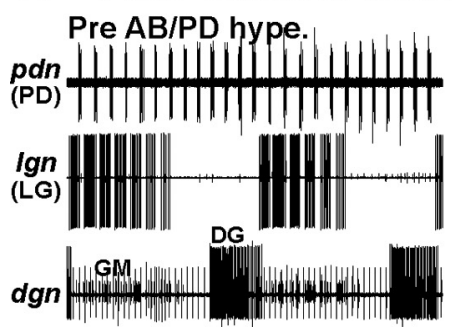

$$
\text { During AB/PD hype. }
$$

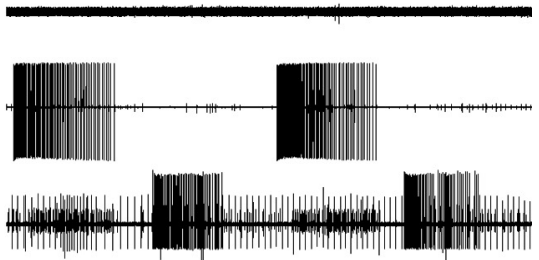

B VCN-Gastric Mill Rhythm
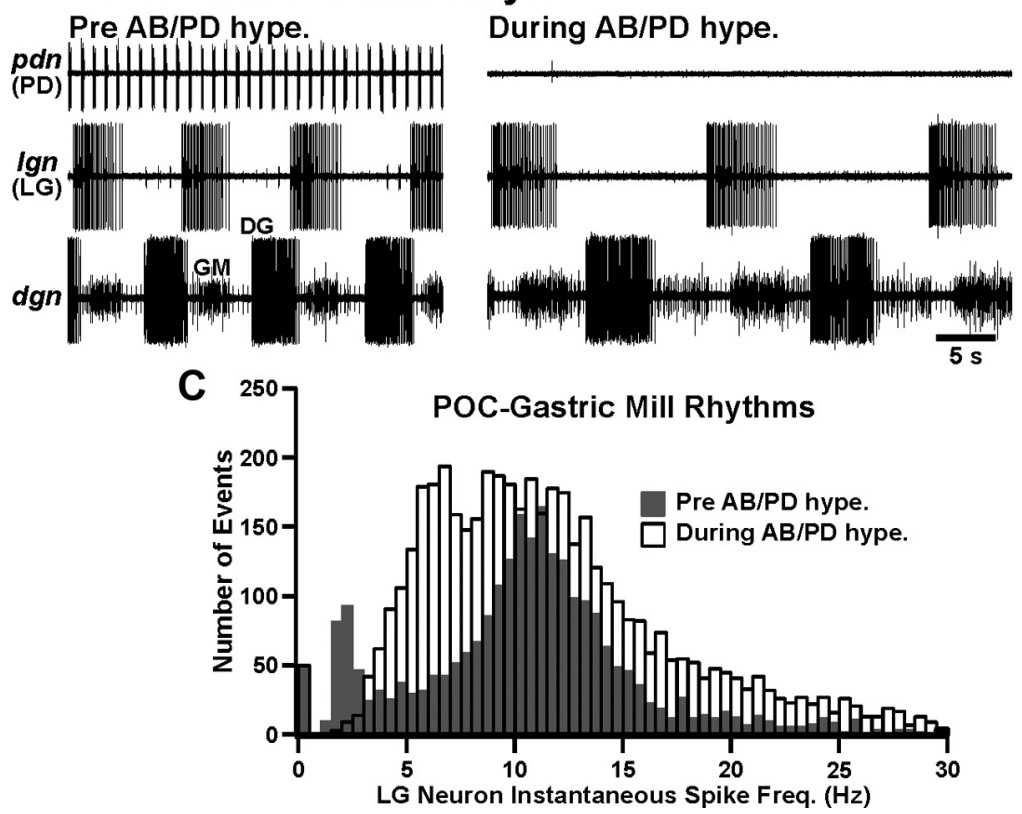

Figure 4. Suppressing the pyloric rhythm did not eliminate the POC- or VCN-gastric mill rhythm but did change the fast rhythmic $L G$ burst pattern to a tonic pattern during the POC rhythm. $A$, The POC-gastric mill rhythm persisted when the pyloric rhythm was suppressed by hyperpolarizing the pyloric pacemaker $(\mathrm{AB} / \mathrm{PD})$ neurons, but it slowed and the pyloric-timed $\mathrm{LG}$ burst pattern (left) was changed to a tonic pattern (right). B, The VCN-gastric mill rhythm persisted, albeit with a longer cycle period, when the pyloric rhythm was suppressed. Note that the $L G$ burst pattern remained tonic in the absence of the pyloric rhythm. $C$, Comparison of the $L G$ instantaneous spike frequency distribution during the POC-gastric mill rhythm in the presence versus absence of the pyloric rhythm. Note that suppressing the pyloric rhythm resulted in an $\sim 12$-fold decrease in the number of events between 1.5 and $3 \mathrm{~Hz}$ (PR on, 235 events; PR off, 19 events). This range included most (91\%: 235 of 259) of the pyloric rhythm (PD neuron)-timed interruptions in the $L G$ burst. The first bar $(0-0.5 \mathrm{~Hz})$ for each condition represents the retractor phase. Bin width is $0.5 \mathrm{~Hz}$. Total number of events: PR on, 2550; PR off, 4702. Data are from five preparations (10 gastric mill cycles per preparation for each condition).

using the paired Student's $t$ test, with the following exceptions. The $\chi^{2}$ goodness-of-fit test (two-tailed) with Yates' correction was used to compare the percentage of pyloric cycles associated with an LG interspike interval pause of at least $200 \mathrm{~ms}$ during the POC- and VCN-gastric mill rhythms. The two-sample Kolmogorov-Smirnov goodness-of-fit test (K-S test) was used to determine the likelihood that the distribution of LG spikes across the pyloric cycle during the POC- and VCN-gastric mill rhythms was the same. As internal controls for the K-S test analysis, we divided each dataset in half and compared them with each other (i.e., half of the POC experiments with the other half of the POC experiments, and the same for the VCN experiments).

In all experiments, the effect of each manipulation was reversible, and there was no significant difference between the pre- and postmanipulation groups. Data are expressed as the mean \pm standard error.

\section{Results}

The VCN- and POC-triggered gastric mill rhythms

The gastric mill rhythm is a two-phase motor pattern that underlies chewing behavior by alternately driving the protraction and retraction of the teeth, which are located in the gastric mill stomach compartment (Heinzel et al., 1993). This rhythm is generated by a CPG circuit in the STG. The gastric mill neurons are all identified and their synaptic interactions characterized (Nusbaum and Beenhakker, 2002; Marder and Bucher 2007; Stein, 2009). This circuit includes four types of protractor motor neurons [LG, GM, medial gastric (MG), inferior cardiac (IC)], three types of retractor motor neurons [DG, ventricular dilator (VD), anterior median $(\mathrm{AM})]$, and a single interneuron (Int1) (Fig. $1 A, B$ ). The motor neurons also have synaptic actions within the circuit (Fig. $1 B$ ), enabling some of them to influence at least some versions of the gastric mill rhythm (Coleman et al., 1995; Bartos et al., 1999; Saideman et al., 2007). The gastric mill rhythm is an episodic motor pattern, in vivo and in vitro, that is driven primarily by projection neurons whose somata are located in the commissural ganglia (CoGs) (Fig. 1A) (Coleman and Nusbaum, 1994; Combes et al., 1999; Beenhakker and Nusbaum, 2004).

In the isolated crab STNS, relatively brief stimulation of the VCN or POC neurons triggers a gastric mill rhythm that commonly persists for tens of minutes after stimulation (Beenhakker et al., 2004; Blitz et al., 2008). The VCNs are a bilateral population $(\sim 60$ neurons per side) of stretch receptor neurons located in the lining of the cardiac sac stomach compartment, a food-storage organ just anterior to the gastric mill compartment (Beenhakker et al., 2004). The VCNs project to the CoGs, in which their activity triggers a long-lasting activation of the projection neurons modulatory commissural neuron 1 (MCN1) and commissural projection neuron 2 (CPN2) (Fig. $1 A-C$ ) (Beenhakker et al., 2004). VCN activation of these two projection neurons is necessary and sufficient to drive the VCN-gastric mill rhythm (Beenhakker and Nusbaum, 2004). All gastric mill neurons participate in the VCN-gastric mill rhythm.

The POCs are a bilateral population of peptidergic neurons $(\sim 100$ per side) that innervate the CoGs via the circumoesophageal commissure $(c o c)$, by which the thoracic ganglion communicates with each CoG (Fig. 1A) (Kirby and Nusbaum, 2007; Blitz et al., 2008). A subset of the POC axons cross to the contralateral coc via the poc (Fig. 1A) (Blitz et al., 2008). Like the VCNs, POC stimulation triggers a long-lasting activation of MCN1 and CPN2, which drives the gastric mill rhythm (Fig. 1B,C) (Blitz et al., 2008). All gastric mill neurons except AM participate in the POC-gastric mill rhythm.

Another distinction between the VCN- and POC-gastric mill motor patterns, besides the AM neuron participating only in the VCN rhythm, is the burst pattern of the protractor neuron LG (Fig. 1C). This neuron commonly fires tonically during the pro- 
traction phase of the VCN-gastric mill rhythm, whereas it exhibits a fast, rhythmic burst pattern during POC protraction. These distinct patterns result from the comparable patterns in MCN1 and CPN2 during each rhythm, because these projection neurons drive LG activity (Fig. 1C) (Coleman and Nusbaum, 1994; Norris et al., 1994; Blitz and Nusbaum, 2008). The fast rhythmic pattern in these projection neurons during POC protraction results from the fast rhythmic feedback inhibition they receive from the pyloric pacemaker interneuron $\mathrm{AB}$ (anterior burster) in the CoGs (Blitz and Nusbaum, 2008). Thus, the fast rhythmic LG burst pattern during the POC-gastric mill rhythm is also pyloric rhythm timed. The tonic pattern in the projection neurons and, thus, in LG during VCN protraction results from the AB feedback being gated out within the CoGs during this time (Blitz and Nusbaum, 2008). Insofar as AB is electrically coupled to and coactive with the paired PD neurons in the STG (Fig. $1 B$ ), the more readily recorded $\mathrm{PD}$ motor neurons serve as a useful monitor of $\mathrm{AB}$ activity (Fig. 1C).

\section{Comparison of the VCN- and POC-triggered gastric mill motor patterns}

Previous studies provided only a qualitative evaluation of the distinct LG burst pattern during the VCN- and POC-gastric mill rhythms. Thus, to more firmly establish this distinction, we analyzed the LG burst structure during each type of gastric mill rhythm. To this end, we determined its within-burst spike distribution relative to the $\mathrm{AB} / \mathrm{PD}$ burst of the pyloric rhythm (see Materials and Methods). When plotted as a function of the phase of the pyloric rhythm, during the POC-gastric mill rhythm, there was a consistent drop in LG activity that began during the PD burst and continued afterward for another $\sim 20 \%$ of each pyloric cycle ( $n=10$ of 10 preparations) (Fig. $2 A, B$ ). In contrast, there was no evident PD-related decline in LG activity during VCNgastric mill rhythms ( $n=10$ of 10 preparations) (Fig. $2 C, D)$. The overall distribution of LG spikes across the pyloric cycle was significantly different during these two gastric mill rhythms $(p=$ $9.2 \times 10^{-5}, n=10$ for each rhythm). In contrast, the distribution of LG spikes across the pyloric cycle was not different when either the POC dataset $(p=0.89, n=10)$ or VCN dataset $(p=0.68, n=$ 10) was divided in half and compared.

We also determined the distribution of these same LG spikes during each pyloric cycle as a function of time instead of pyloric phase, by binning these spikes $(10 \mathrm{~ms} / \mathrm{bin} ; \sim 1 \%$ of the pyloric cycle), starting with PD burst onset. With this approach, during the POC-gastric mill rhythm, the biggest decline in the PD-timed activity of LG, wherein each bin contained $\leq 0.2 \%$ of the total LG spikes during the pyloric cycle, commonly lasted for at least 200 ms. We therefore determined the fraction of PD neuron bursts during each LG burst that were associated with a pause in LG firing of at least $200 \mathrm{~ms}$. During the POC-gastric mill rhythm, there were such pauses in LG activity during $90 \%$ of all pyloric cycles ( 404 of 448 pyloric cycles, $n=10$ preparations). Additionally, in all of the remaining 44 cycles, a briefer, PD-timed pause was still evident in LG activity. In contrast, during the VCNgastric mill rhythm, comparable pauses of at least $200 \mathrm{~ms}$ in LG activity occurred in a significantly smaller percentage of pyloric cycles (5\%: 23 of 466 pyloric cycles, $n=10$ preparations; $p<$ 0.0001 relative to the POC-gastric mill rhythm). Furthermore, in every case in which this pause did occur during the VCN rhythm ( $n=23$ of 23), there was only one per LG burst, and it took place at the end of the burst when the LG firing rate was waning.

We also compared several parameters that define the gastric mill motor pattern and found additional distinctions between the
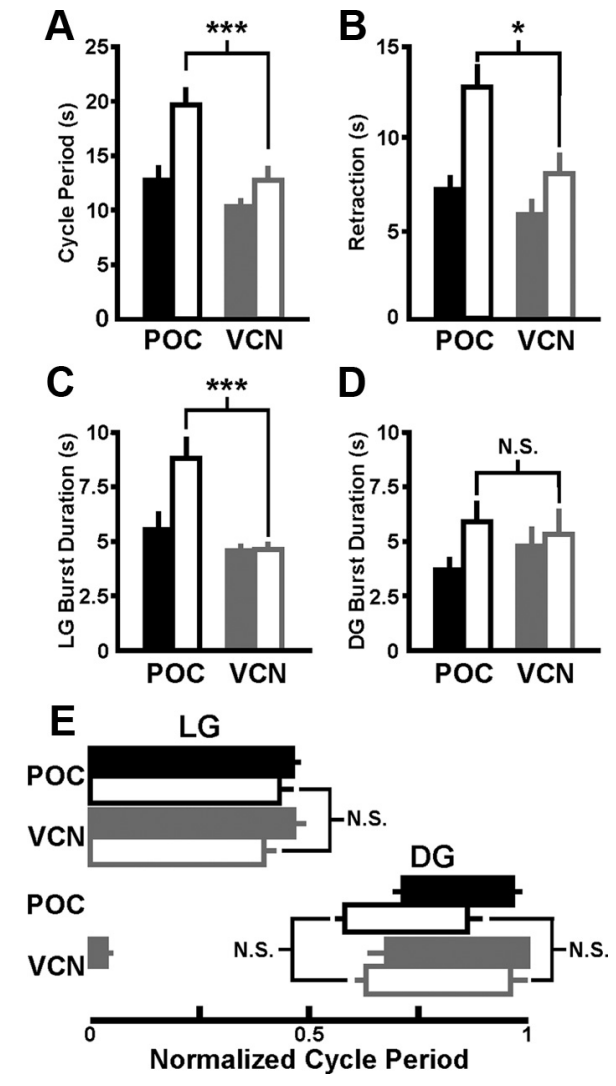

Figure 5. Suppressing the pyloric rhythm did not eliminate all differences between the POCand VCN-gastric mill motor patterns. $\boldsymbol{A}-\boldsymbol{C}$, The POC- and VCN-gastric mill cycle period $(\boldsymbol{A})$, retraction burst duration $(\boldsymbol{B})$, and protraction (LG burst) duration $(\boldsymbol{C})$ remained distinct when the pyloric rhythm was suppressed. $D$, The DG burst duration remained comparable during the POC- and VCN-gastric mill rhythms when the pyloric rhythm was suppressed. $\boldsymbol{E}$, The LG burst offset phase remained comparable, whereas that of the DG neuron became comparable during the POC- and VCN-gastric mill motor patterns when the pyloric rhythm was suppressed. POC rhythm, $n=8$; VCN rhythm, $n=8$. Filled bars, Pyloric rhythm active; open bars, pyloric rhythm suppressed; ${ }^{*} p<0.05 ;{ }^{* * *} p<0.001$; N.S., not significant $(p>0.05)$.

VCN- and POC-gastric mill rhythms. One such distinction was that the cycle period was briefer during the VCN-gastric mill rhythm (VCN, $10.36 \pm 0.4 \mathrm{~s}, n=10$; POC, $13.39 \mathrm{~s} \pm 1.1 \mathrm{~s}, n=10$; $p=0.02$ ) (Fig. 3A). The longer cycle period for the POC rhythm resulted from a prolongation of both the protractor phase (i.e., LG burst duration) (VCN, $4.47 \pm 0.2 \mathrm{~s}, n=10$; POC, $5.61 \pm 0.5 \mathrm{~s}$, $n=10 ; p=0.04)$ and the retractor phase (VCN, $5.89 \pm 0.3 \mathrm{~s}, n=$ 10; POC, $7.82 \pm 0.7$ s, $n=10 ; p=0.03$ ) (Fig. $3 A, B$ ).

In parallel with the increased protractor phase duration during the POC-gastric mill rhythm, the protractor neurons GM (POC, $n=10$; VCN, $n=10, p=0.02$ ) and MG (POC, $n=10$; $\mathrm{VCN}, n=10 ; p=0.03$ ) exhibited longer-duration bursts during this version of the gastric mill rhythm (Fig. $3 B$ ). In contrast, despite the longer retractor phase duration during the POC rhythm, the retractor motor neuron DG burst duration was not different during the POC and VCN rhythms (each rhythm: $n=$ $10, p=0.98$ ) (Fig. 3B). During both rhythms, the DG burst began partway through the retractor phase and terminated near the time of $\mathrm{LG}$ burst onset (Figs. 1C, 3C). Last, there was no difference in the duration of the gastropyloric motor neuron IC and VD bursts (each rhythm: $n=10, p=0.3$ ) (Fig. $3 B$ ). IC and VD activity spanned the retractor phase and overlapped the initial part of protraction (Figs. $1 C, 3 C$ ).

There were also some differences in duty cycle and phase relationships during the VCN and POC rhythms. Specifically, the 

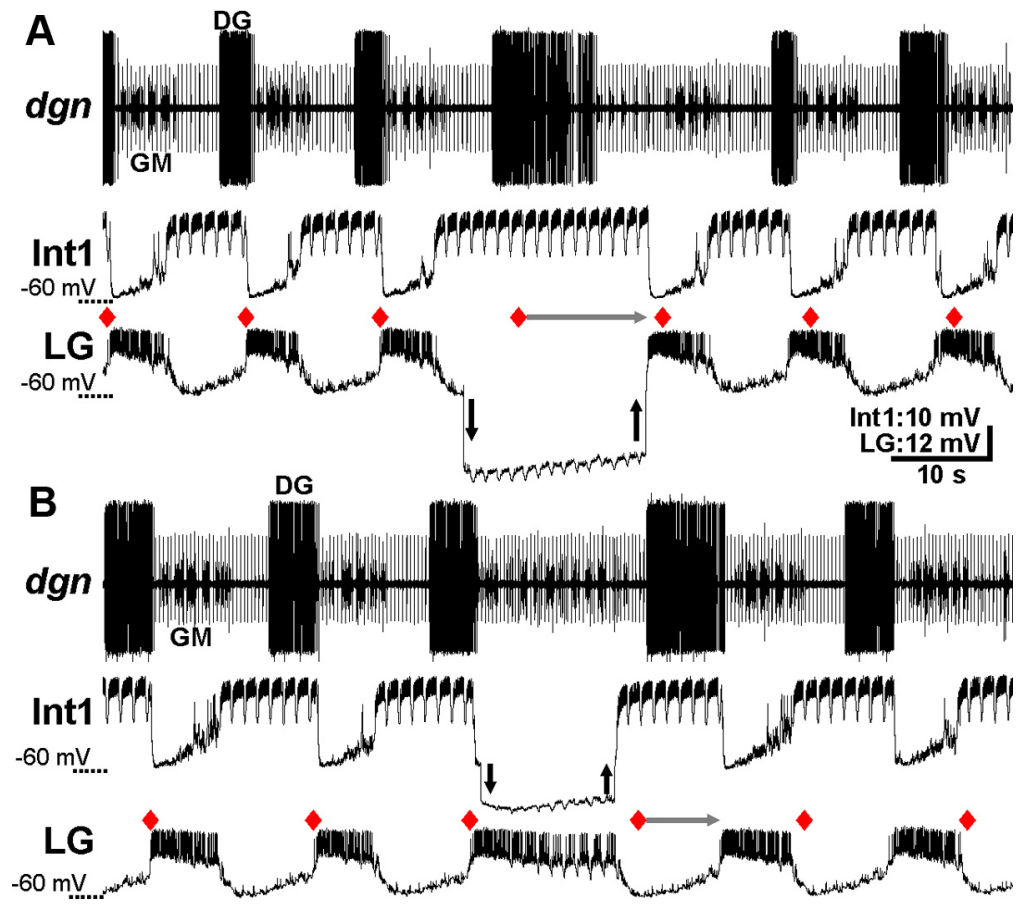

Figure 6. $\quad \mathrm{LG}$ and Int1 are necessary for VCN-gastric mill rhythm generation. $A$, During a VCN-gastric mill rhythm, the $L G$ neuron was hyperpolarized (arrows) for longer than its gastric mill rhythm-timed inhibition by Int1. Red diamonds indicate the expected LG burst onset, based on the five successive gastric mill cycles before the LG hyperpolarization. Note that, during the LG hyperpolarization, Int1 did not exhibit its anticipated, protractor phase-associated hyperpolarization starting at the red diamond, as should have occurred if the gastric mill rhythm was not influenced by suppressing LG activity. As indicated by the horizontal gray arrow, the next Int1 hyperpolarization, and associated LG burst, was delayed until after the LG hyperpolarization. Note also that the DG burst duration was prolonged but not for the duration of the LG hyperpolarization. Downward and upward arrows indicate the start and end of hyperpolarizing current injection, respectively. B, During a VCN-gastric mill rhythm, suppressing Int1 activity by hyperpolarizing current injection (arrows) for longer than the duration of its inhibition by $L G$ delayed the $L G$ burst termination until after the period of hyperpolarization. Thus, the next gastric mill cycle onset (i.e., LG burst onset: gray arrow) after the start of Int1 hyperpolarization was delayed until well after the period of hyperpolarization. Both panels are from the same preparation.

retractor neuron DG duty cycle was larger during the VCN rhythm (each rhythm; $n=10, p=0.01$ ), whereas the duty cycle of the protractor neuron MG was larger during the POC rhythm (each rhythm, $n=10, p=0.049$ ). With respect to phase, relative to its burst onset during the POC rhythm, during the VCN rhythm the burst onset of the protractor GM (each rhythm: $n=$ $10, p=0.0001$ ) and MG (each rhythm: $n=10, p=0.009$ ) neurons were consistently phase delayed (Fig. $3 C$ ). In contrast, DG burst offset was phase delayed during the VCN rhythm (each rhythm: $n=10, p=0.02$ ) (Fig. $3 C$ ).

Thus, the POC-and VCN-gastric mill rhythms were distinct with respect to their cycle periods and many aspects of their patterns. The most prominent distinctions spanned five of the eight types of gastric mill neurons, including AM neuron participation only during the VCN-gastric mill rhythm and the distinct LG neuron burst structure, DG neuron duty cycle, and burst onset phase of the GM and MG neurons.

Influence of the pyloric rhythm on the VCN- and POCtriggered gastric mill motor patterns

One clear distinction between the VCN- and POC-gastric mill rhythms is the relative influence of the pyloric rhythm on the projection neurons $\mathrm{MCN} 1$ and $\mathrm{CPN} 2$ during the gastric mill protractor phase (Blitz and Nusbaum, 2008) and the resulting distinction in the LG burst pattern during these two motor patterns (Figs. 1C, 2). Thus, we tested the hypothesis that all of the identified differences between these two gastric mill motor patterns resulted from this distinct influence of the pyloric rhythm. To this end, we compared VCN- and POC-gastric mill motor patterns in preparations in which we suppressed the pyloric rhythm by injecting constant amplitude hyperpolarizing current into the pyloric pacemaker neurons ( $\mathrm{AB}$ and $\mathrm{PD}$ neurons). We continuously monitored the pyloric rhythm by extracellular recordings of all pyloric motor neurons, including the PD neurons.

As shown previously for the POC rhythm (Blitz et al., 2008), suppressing the pyloric rhythm did not terminate either type of gastric mill rhythm (POC rhythm, $n=8$; VCN rhythm, $n=8$ ) (Fig. $4 A, B$ ). Thus, the pyloric rhythm was not necessary for POC- or VCN-gastric mill rhythm generation. However, as anticipated, suppressing the pyloric rhythm did alter these gastric mill motor patterns. For example, for the POC-gastric mill rhythm, suppressing the pyloric rhythm increased the cycle period [pyloric rhythm (PR) on, $12.7 \pm 1.2 \mathrm{~s}$; PR off, $19.6 \pm 1.7 \mathrm{~s}$; $n=8, p<0.0001$ ], protraction (LG burst) duration (PR on, $5.6 \pm 0.7 \mathrm{~s}$; PR off, $8.8 \pm$ 0.9 s; $n=8, p<0.0001)$, and retraction (LG interburst) duration (PR on, $7.1 \pm$ $0.7 \mathrm{~s} ;$ PR off, $12.8 \pm 1.4 \mathrm{~s} ; n=8, p<$ $0.001)$. In contrast, for the VCN-gastric mill rhythm, suppressing the pyloric rhythm did not change either the cycle period (PR on, $10.3 \pm 0.6 \mathrm{~s}$; PR off, $12.7 \pm$ $1.1 \mathrm{~s} ; n=8, p=0.06$ ) or protraction duration (PR on, $4.6 \pm 0.3 \mathrm{~s}$; PR off, $4.6 \pm$ $0.2 \mathrm{~s} ; n=8, p=0.41$ ), but it did prolong retraction (PR on, $5.7 \pm$ $0.7 \mathrm{~s} ;$ PR off, $8.0 \pm 1.0 \mathrm{~s} ; n=8, p=0.04$ ).

Suppressing the pyloric rhythm also altered the burst structure of those gastric mill neurons whose gastric mill rhythmrelated burst normally exhibited pyloric-timed interruptions in firing. For example, as first reported qualitatively by Blitz et al. (2008), the pyloric-timed LG burst pattern during the POC rhythm changed to a tonic pattern when the pyloric rhythm was suppressed (eight of eight preparations) (Fig. $4 A, C$ ). In general, during these tonic LG bursts, the first half of each burst exhibited higher-frequency firing than the latter half (Fig. $4 A$ ). The pattern change in LG when the pyloric rhythm was suppressed during the POC-gastric mill rhythm included an $\sim 12$-fold decrease in the number of events (PR on, 235; PR off, 19) within the instantaneous spike frequency range (1.5-3 $\mathrm{Hz}$ ) in which most pyloric-timed interruptions in LG activity occurred (235 of 259: 91\%; $n=5$ ) when the pyloric rhythm was active (Fig. 4C). This reduced number of events between 1.5 and $3 \mathrm{~Hz}$ when the pyloric rhythm was suppressed occurred despite the considerably larger total number of events during this condition, as a result of the LG burst pattern switch from fast rhythmic to tonic firing and the prolonged LG burst duration (PR on, 2550 events; PR off, 4702 events; $n=5$ preparations, 50 cycles for each condition). Suppressing the pyloric rhythm also broadened considerably the peak distribution of LG instantaneous spike frequencies (Fig. 4C). 
Although the LG intraburst structure was changed by suppressing the pyloric rhythm during the POC-gastric mill rhythm, its mean intraburst firing frequency (excluding the pyloric-timed interruptions) did not change (PR on, $8.8 \pm 1.0 \mathrm{~Hz}$; PR off, $11.0 \pm 1.4 \mathrm{~Hz}$; $n=8, p=0.08$ ). However, the combination of the increased LG burst duration (see above) and the elimination of pyloric-timed interruptions more than doubled the mean number of LG spikes per burst when the pyloric rhythm was suppressed (PR on, $44.8 \pm$ 7.5 spikes; PR off, $96.5 \pm 13.4$ spikes; $n=8, p<0.001)$. The LG burst termination during the POC rhythm was also slightly phase advanced when the pyloric rhythm was suppressed (PR on, $0.43 \pm 0.03 ;$ PR off, $0.41 \pm 0.04 ; n=8, p=0.04)$. In contrast to the increased number of LG spikes per burst during the POC rhythm resulting from pyloric rhythm suppression, the same manipulation during the VCN-gastric mill rhythm did not alter this parameter (PR on, $51.3 \pm 7.5$ spikes; PR off, $56.3 \pm 8.8$ spikes; $n=8, p=0.09$ ) nor, as reported above, did it change the LG burst duration. However, as during the POC rhythm (see above), the LG burst termination during the VCN rhythm was phase advanced by suppressing the pyloric rhythm (PR on, $0.49 \pm 0.04$; PR off, $0.38 \pm 0.04 ; n=8, p=0.02$ ).

Suppressing the pyloric rhythm also changed the burst pattern from fast rhythmic to tonic for the GM neurons during the POCgastric mill rhythm (Fig. 4A) and during both rhythms for the Int1, MG, IC, and VD neurons (data not shown). Thus, with the pyloric rhythm suppressed during the VCN- and POC-gastric mill rhythms, the burst structure of many gastric mill neurons converged to a tonic bursting pattern.

Despite the overall convergence of the gastric mill neuron burst structures to a tonic firing pattern when the pyloric rhythm was suppressed, comparing the VCN- and POC-gastric mill motor patterns during this manipulation showed that these patterns remained distinct with respect to other parameters. This was the case, for example, for the gastric mill cycle period, protraction duration, and retraction duration (Fig. $5 A-C$ ). There was little change in the level of significant difference for the cycle period and retraction duration relative to these values when the pyloric rhythm was active (Figs. $3 A, 5 A, B$ ), but the protraction duration exhibited an increased significant difference when the pyloric rhythm was suppressed (Figs. 3B, 5C). Additionally, one parameter that was comparable during both gastric mill rhythms with the pyloric rhythm active, the number of LG spikes/burst, diverged when the pyloric rhythm was suppressed ( $\mathrm{PR}$ on, $p=0.16$; PR off, $p=0.03$ ). Some parameters that had been distinct between these two gastric mill rhythms did become comparable when the pyloric rhythm was suppressed, such as the DG duty cycle (PR on, $p=0.01 ; \mathrm{PR}$ off, $p=0.26$ ) and the phase of its burst termination (PR on, $p=0.02$; PR off, $p=0.28$ ) (Figs. $3 C, 5 D, E$ ). Overall, although the pyloric rhythm was responsible for the fast rhythmic burst pattern in many gastric mill neurons, it was not the source of all the differences in the gastric mill rhythm parameters that distinguished the POC- and VCN-gastric mill motor patterns.

\section{Identifying the core rhythm-generating neurons during the VCN- and POC-gastric mill rhythms}

The core rhythm-generating neurons for the version of the gastric mill motor pattern driven by tonic MCN1 stimulation in reduced preparations, with the CoGs removed, include LG and Int1 (Coleman et al., 1995; Bartos et al., 1999; Saideman et al., 2007). The same gastric mill motor pattern is elicited by bath applying Cancer borealis pyrokinin (CabPK) peptide, again in the isolated STG, although in this latter condition the core rhythm generator includes LG, Int1, and AB (Saideman et al., 2007). This version of

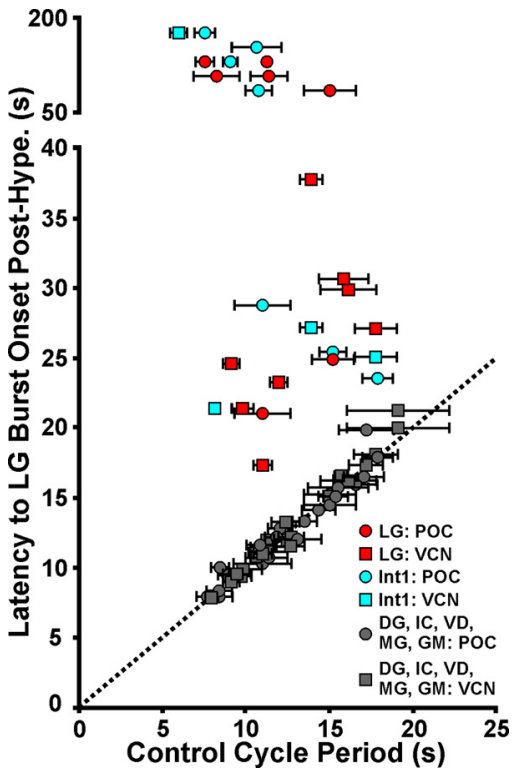

Figure 7. LG and Int1, but no other gastric mill circuit neuron, are necessary for POC- and VCN-gastric mill rhythm generation. Reversibly hyperpolarizing either LG or Int1 during the VCN-or POC-gastric mill rhythm consistently delayed the onset of the next gastric mill cycle until after the period of imposed hyperpolarization. In contrast, hyperpolarizing any of the other gastric mill neurons did not alter the gastric mill cycle period. Each data point represents the results of a single hyperpolarizing current duration in a single preparation. For all neurons, the hyperpolarizing current injection duration, in different preparations, ranged from 10 to $180 \mathrm{~s}$. For the LG and Int1 manipulations, the first LG burst onset after the current injection was terminated occurred either as soon as the injected current was removed (LG injections) or within 5-10 s (Int1 injections). Number of different preparations per neuron: POC-gastric mill rhythm: LG, 7; Int1, 7; MG, 3; IC, 7; GM, 2; VD, 5; DG, 7; VCN-gastric mill rhythm: LG, 8; Int1, 5; MG, 3; IC, $4 ; G M, 3 ; V D: 3 ; D G, 6$. Dotted line: slope $=1$. Data points on the dotted line indicate equivalent values on the $x$ - and $y$-axis.

the gastric mill motor pattern is distinct from those triggered by VCN or POC stimulation. For example, neither the GM nor AM neurons participate in the MCN1/CabPK-elicited gastric mill rhythm, the VD neuron is active only during retraction, and the IC neuron is predominantly active during protraction (Saideman et al., 2007). We therefore aimed to determine whether LG and Int 1 also comprised the core rhythm generator for the gastric mill rhythms triggered by VCN and POC stimulation. To this end, we selectively and reversibly suppressed activity in each gastric mill neuron during VCN and POC rhythms for durations that were longer than their normal interburst duration and determined whether doing so interfered with the ongoing rhythm (see Materials and Methods).

Transiently hyperpolarizing either LG $(n=8)$ or Int1 $(n=5)$ consistently and reversibly disrupted the VCN-gastric mill rhythm. For example, as shown in Figure 6, the gastric mill cycle period was regular from cycle-to-cycle before each hyperpolarization. In contrast, during the maintained LG or Int1 hyperpolarization, the start of the next gastric mill cycle did not occur at the anticipated time. Instead, the next cycle onset was consistently delayed until sometime after the hyperpolarizing current injection was removed (cycle period: LG control, $13.2 \pm 1.2 \mathrm{~s}$; LG hyperpolarized, $26.5 \pm 2.4 \mathrm{~s} ; p=0.0001, n=8$; Int1 control, $11.5 \pm 2.7 \mathrm{~s}$; Int1 hyperpolarized, $23.4 \pm 1.6 \mathrm{~s} ; p=0.002, n=5$ ) (Figs. 6, 7). Resumption of the gastric mill rhythm always began with a burst in the previously hyperpolarized neuron (Fig. 6). Moreover, after each hyperpolarizing current injection, the rhythm was reset in that the start of each subsequent gastric mill cycle (i.e., LG burst onset) did not return to occurring at its expected 


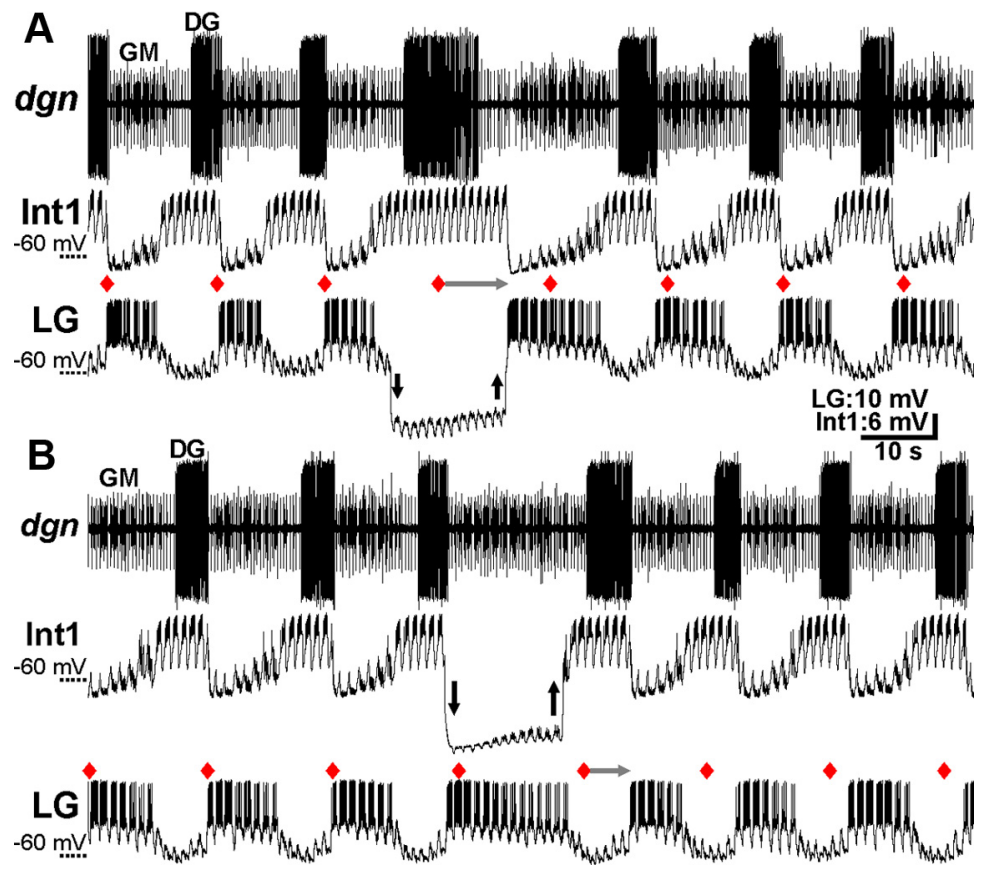

Figure 8. $L G$ and Int1 are necessary for POC-gastric mill rhythm generation. $A$, During a POC-gastric mill rhythm, hyperpolarizing the $L G$ neuron (arrows) for longer than the duration of its inhibition by Int1 delayed the start of the next episode of protraction-related Int 1 hyperpolarization (i.e., start of the next gastric mill cycle) until after the current injection (gray arrow). Int1 remained active for the entire LG hyperpolarization, and the DG burst duration was also prolonged. The next expected gastric mill cycle onset (i.e., LG burst onset) after the start of hyperpolarizing current injection, in the absence of that current injection, is indicated by the red diamond. $\boldsymbol{B}$, During a POC-gastric mill rhythm, suppressing Int1 activity by hyperpolarizing current injection (arrows) for longer than its inhibition by LG delayed the start of the next gastric mill cycle until after the period of current injection (gray arrow). The start of the next anticipated gastric mill cycle (i.e., LG burst onset) after the beginning of current injection into Int1 is indicated by the red diamond. Both panels are from the same preparation.

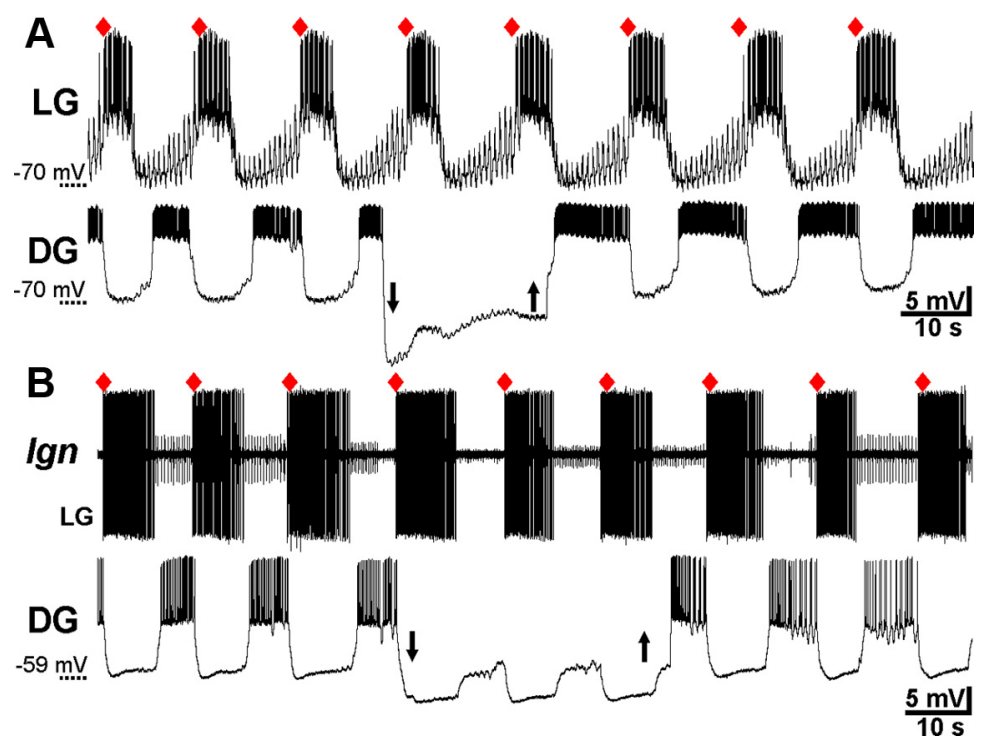

Figure 9. $D G$ is not necessary for POC- or VCN-gastric mill rhythm generation. A, During a POC-gastric mill rhythm, DG was hyperpolarized (arrows) for a duration that was longer than its normal gastric mill interburst period, but doing so did not delay the next expected $L G$ burst onset (red diamond). B, Suppressing DG activity with hyperpolarizing current injection (arrows) did not delay the next expected $\mathrm{LG}$ burst onset during a VCN-gastric mill rhythm. $\boldsymbol{A}$ and $\boldsymbol{B}$ are from different preparations. whereas the retractor phase neuron Int1 did consistently maintain its retractor activity pattern, the retractor DG motor neuron burst was prolonged but not for the duration of the LG hyperpolarization $(n=8$ of 8 ) (Figs. 6A, 8A). Additionally, the protractor phase neurons exhibited relatively weak activity during prolonged LG hyperpolarizations (Fig. 6A). Similarly, the protractor phase was not well maintained during Int1 hyperpolarization. For example, the LG activity waned over time ( $n=5$ of 5 ) (Fig. $6 B)$. The retractor neurons, such as DG, were weakly active or silent (Figs. 6B, 8B). The disruption and subsequent resumption of the ongoing motor pattern occurred consistently across the $\sim 10$-fold range of current injection durations used for LG and Int1 (Fig. 7).

Given the pivotal influence of LG and Int 1 on rhythm generation during the VCN- and POC-motor patterns, we determined whether there was a difference in the range of their membrane potential oscillations during each rhythm, insofar as it might contribute to the differences between these two motor patterns. Across preparations, the slow wave membrane potential in LG was comparable during both motor patterns at the peak $(\mathrm{VCN}$, $-39.0 \pm 1.2 \mathrm{mV}, n=6$; POC, $-39.6 \pm$ $1.5 \mathrm{mV} ; n=7, p=0.09)$ and trough (VCN, $-63.0 \pm 0.8 \mathrm{mV}, n=6$; POC, $-61.3 \pm 0.1 \mathrm{mV} ; n=7, p=0.4)$ of its gastric mill-timed profile. This was also the case for the Int 1 peak $(\mathrm{VCN},-37.7 \pm$ $4.8 \mathrm{mV}, n=4$; POC, $-43.4 \pm 3.6 \mathrm{mV}, n=$ $6 ; p=0.2)$ and trough $(\mathrm{VCN},-63.2 \pm 2.0$ $\mathrm{mV}, n=4$; POC, $-62.4 \pm 2.9 \mathrm{mV}, n=6$; $p=0.4$ ) membrane potentials.

In contrast to the ability of LG and Int1 to influence gastric mill rhythm generation after VCN or POC stimulation, reversibly suppressing the activity of any one of the other gastric mill neurons never altered these ongoing rhythms, regardless of the duration of the hyperpolarizing current injection ( $p>0.05$ for all six neuron types; $n=$ $3-10$, both gastric mill rhythms) (Fig. 7). One example of this result is shown in Figure 9 for hyperpolarization of the retractor neuron DG. In neither the VCN- nor POCgastric mill rhythm did suppressing DG activity alter the expected onset time of the next gastric mill cycle. This result was not necessarily a foregone conclusion, because the DG neuron does influence the gastric onset time in the absence of current injection (Fig. 6). The same results were obtained when LG or Int 1 was reversibly hyperpolarized during the POC-gastric mill rhythm (LG, $p=0.002, n=6$; Int $1, p=$ $0.017, n=7$ ) (Figs. 7, 8).

When LG activity was suppressed by hyperpolarizing current injection, the retractor phase was not simply prolonged. For example, mill rhythm activated by bath applying CabPK (Saideman et al., 2007).

\section{Discussion}

In this paper, we have shown that the same core rhythm generator underlies different versions of a rhythmic motor pattern trig- 


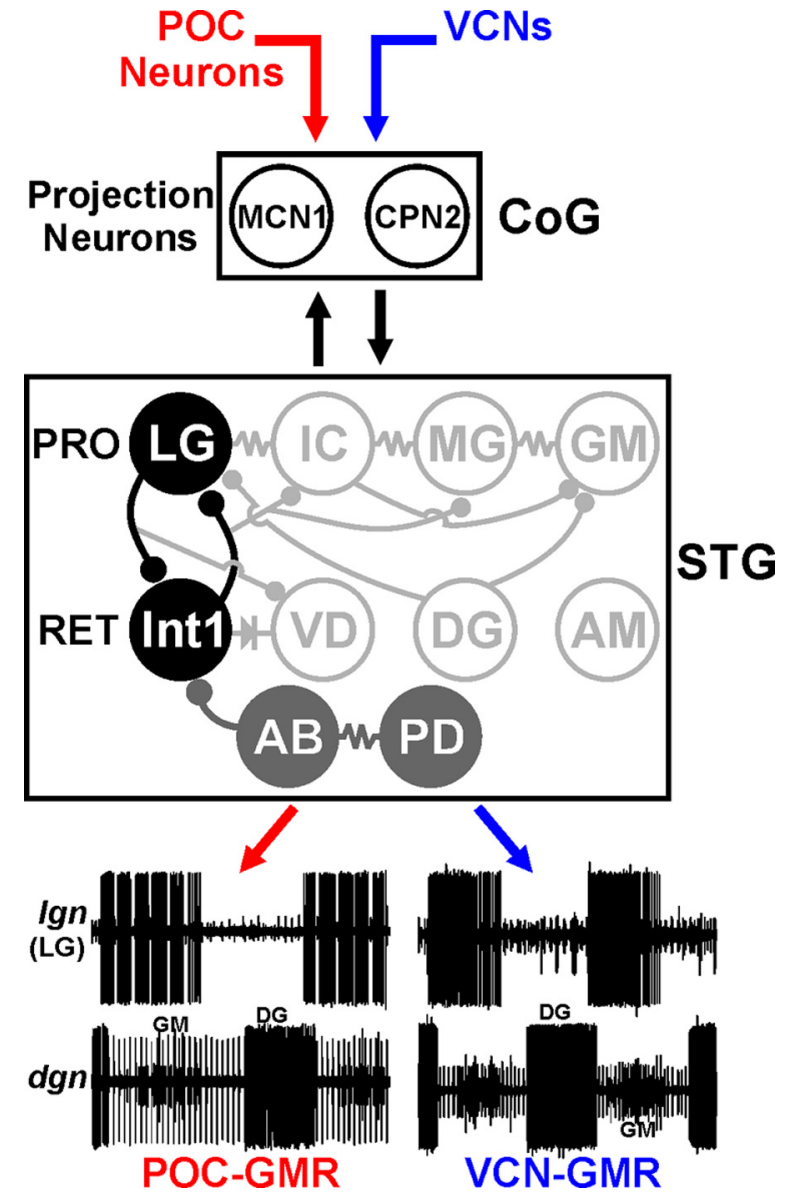

Figure 10. The gastric mill circuit neurons $L G$ and $\operatorname{lnt} 1 \mathrm{form}$ the core rhythm generator for the POC- and VCN-gastric mill rhythms. Two different extrinsic inputs, POC- and VCN neurons, trigger different gastric mill motor patterns by activating the same CoG projection neurons (MCN1, CPN2). LG and Int 1 are the only gastric mill neurons necessary for generating the POCand VCN-gastric mill rhythms. The pyloric pacemaker neurons (AB, PD), however, regulate the cycle period and pattern of both gastric mill rhythms. Hence, in parallel with their pivotal role in generating the pyloric rhythm, $A B$ and $P D$ are pattern generator neurons for these gastric mill rhythms. The POC- and VCN-gastric mill rhythms (GMRs) are represented by extracellular recordings of the LG (Ign) and GM (dgn, small units) protractor (PRO) neurons firing their rhythmic bursts in alternation with those of the DG retractor (RET) neuron (dgn, large unit). Note the fast rhythmic $L G$ burst pattern during the POC-GMR and its tonic burst pattern during the VCNGMR. The pyloric pacemaker neuron inhibition of other gastric mill neurons (VD, IC, MG) is omitted for clarity. STG neurons: Black filled circles, Core POC- and VCN-gastric mill rhythm generator neurons; gray filled circles, POC- and VCN-gastric mill pattern generator neurons; open circles, gastric mill follower motor neurons. Synapse symbols as in Figure 1.

gered by different input pathways. Specifically, the reciprocally inhibitory neurons LG and Int1 are the only gastric mill circuit neurons necessary for rhythm generation during the distinct, VCN- and POC-triggered gastric mill rhythms in the crab $C$. borealis (Fig. 10). It is not a foregone conclusion that different motor patterns generated by the same motor circuit would have the same rhythm generator. One reason for this uncertainty is that, for many CPGs, the different motor patterns they generate often result at least partly from a change in the set of participating neurons (Jing and Weiss, 2002; Popescu and Frost, 2002; Proekt et al., 2007; Briggman and Kristan, 2008; Berkowitz et al., 2010; Weaver et al., 2010). In contrast, one might anticipate that the same motor pattern elicited by different inputs would be driven by the same core rhythm generator, yet distinct albeit overlapping sets of neurons are necessary for generating the comparable MCN1- and CabPK-elicited gastric mill motor patterns. Specifi- cally, they both include LG and Int1, but the pyloric pacemaker neuron $\mathrm{AB}$ is also necessary for CabPK-gastric mill rhythm generation (Coleman et al., 1995; Bartos et al., 1999; Saideman et al., 2007). The gastric mill motor pattern activated by MCN1 and CabPK is also distinct from the ones triggered by the POC and VCN pathways (Saideman et al., 2007).

The basis of rhythm generation in CPGs is classically separated into networks paced by intrinsically oscillatory neurons, often called pacemaker-driven CPGs, and those in which rhythm generation results from a combination of non-oscillatory intrinsic properties and synaptic interactions (network-driven CPGs) (Marder and Bucher, 2001; Marder et al., 2005; Selverston 2010). A common synaptic interaction motif in network-driven CPGs is reciprocal inhibition, as between the LG neuron and Int1. Some or all of the rhythm-generating neurons for at least one version of a rhythmic motor pattern are identified in a number of pacemakerdriven and network-driven rhythmic motor systems (Selverston and Miller, 1980; Getting and Dekin, 1985; Masino and Calabrese, 2002; Cangiano and Grillner, 2003, 2005; Katz et al., 2004; Peña et al., 2004; Pirtle and Satterlie, 2006; Saideman et al., 2007; Li et al., 2010; Selverston, 2010).

With respect to the degree of preservation of the core rhythm generator when a CPG produces different motor patterns, the pacemaker-driven pyloric circuit is the most extensively studied. Under the different modulatory conditions in which the pyloric rhythm generator has been identified, the pyloric pacemaker group (AB, PDs) retains this role (Hooper and Marder, 1987; Ayali and Harris-Warrick, 1999; Marder and Bucher, 2007). Similarly, the pacemaker-driven timing network for leech heartbeat is unchanged when each side of the system reciprocally switches its pattern between peristaltic and synchronous modes (Masino and Calabrese, 2002; Weaver et al., 2010). In contrast, work in the mammalian respiratory system suggests that its core rhythm generator switches between different types of pacemaker neurons during different respiratory behaviors (Peña et al., 2004). Less is known regarding preservation of the rhythm generator during different versions of a network-driven motor pattern. As discussed above, for the network-driven crab gastric mill CPG, the core rhythm generator group had been identified for one gastric mill motor pattern, driven by either tonic MCN1 stimulation or bath-applied CabPK (Saideman et al., 2007). Our current work establishes that the gastric mill rhythm generator can persist during different versions of this motor pattern. Although the number of systems studied remains limited, it appears that the neurons contributing to the core rhythm generator for a particular motor system can either persist or be modified when different versions of the motor pattern are elicited. This provisional conclusion suggests that this feature has more flexibility than other, more extensively characterized general principles of CPG organization (Marder and Calabrese, 1996; Marder and Bucher, 2001; Selverston, 2010).

Different versions of a particular motor pattern commonly result either from modulating the properties of the same set of pattern generating neurons or altering the set responsible for pattern generation (Marder et al., 2005; Marder and Bucher, 2007; Briggman and Kristan, 2008; Sasaki et al., 2009; Berkowitz et al., 2010). In contrast, the distinctions between the VCN- and POC-gastric mill rhythms appear to result at least partly from a selective gating-out of the feedback inhibition from the pyloric pacemaker neuron $\mathrm{AB}$ to $\mathrm{MCN} 1$ and $\mathrm{CPN} 2$ during the VCNgastric mill protractor phase (Blitz and Nusbaum, 2008). This gating mechanism underlies the tonic versus pyloric-timed activity of MCN1 and CPN2 during the VCN- and POC-gastric mill 
protraction phase, respectively, which in turn determines the LG activity pattern. There must, however, be additional differences mediated by the POC and VCN pathways, insofar as the two gastric mill rhythms remained distinct in at least several respects when the pyloric rhythm was suppressed.

The fact that LG and Int1 were the only gastric mill neurons necessary for generating the VCN- and POC-gastric mill rhythms does not necessarily mean that other gastric mill neurons cannot influence rhythm generation. For example, the other protractor motor neurons are electrically coupled to LG. Consequently, whereas individual manipulations of these neurons did not interfere with the ongoing rhythm, coincident membrane potential changes in several of these neurons might produce such a change. Within the pyloric pacemaker group, electrical coupling enables the paired $\mathrm{PD}$ neurons to regulate the cycle period of the intrinsically oscillatory $\mathrm{AB}$ neuron, and manipulating both $\mathrm{PD}$ neurons has a stronger influence than either one alone on the $A B$ cycle period (Hooper and Marder, 1987; Ayali and Harris-Warrick, 1999).

The fact that the POC- and VCN-gastric mill motor patterns were both altered by suppressing the pyloric rhythm indicates that the pyloric pacemaker neurons are pattern generator neurons for the gastric mill rhythm, in parallel with their well established roles as rhythm generator and pattern generator neurons for the pyloric rhythm (Marder and Bucher, 2007). For example, suppressing $\mathrm{AB}$ and $\mathrm{PD}$ neuron activity switched the activity pattern of all gastric mill neurons that normally exhibit pylorictimed activity during the VCN- and POC-gastric mill rhythms to a tonic bursting pattern. This pattern change in the gastric mill motor neurons will likely influence both the pattern and strength of contraction of the muscles that they innervate (Heinzel et al., 1993; Stein et al., 2006; White et al., 2007). Earlier work by Weimann and Marder (1984), using gastric mill rhythms elicited by bath-applied modulators, drew the similar conclusion that current injection into some pyloric neurons could reset the gastric mill cycle period as could some gastric mill neurons for the pyloric cycle period. This observation adds to the previously established, intertwined nature of the gastric mill and pyloric circuits, which exhibit coordinated activity and regulate each other's cycle period, despite functioning with mean cycle periods that are $\sim 10$-fold different (Bartos and Nusbaum, 1997; Clemens et al., 1998; Nadim et al., 1998; Bartos et al., 1999; Thuma and Hooper, 2002; Wood et al., 2004; Bucher et al., 2006). Many complex behaviors involve coordination between separate motor networks, as occurs for example between locomotion and respiration (Kawahara et al., 1989; Syed and Winlow, 1991; Bernasconi and Kohl, 1993; Morin and Viala, 2002; Saunders et al., 2004; Gariépy et al., 2010). Thus far, however, in most of these systems, it remains to be determined whether the coordination results from interactions between the two CPGs or is imposed on them from descending and/or ascending inputs (Ezure and Tanaka, 1997; Morin and Viala, 2002; Steriade, 2006).

Whether there are separate conditions in vivo that selectively activate the POC or VCN pathway to drive their two distinct gastric mill motor patterns is not yet known, although VCN-like gastric mill rhythms have been recorded in vivo (Heinzel et al., 1993). However, in vivo endoscope analysis has shown that the LG neuron-driven lateral teeth protract either smoothly or in a pyloric-timed pattern, supporting a natural behavioral role for the VCN- and POC-gastric mill patterns (Heinzel et al., 1993). As methodological developments for in vivo recordings and manipulations continue to be refined (Hedrich et al., 2011), it will become possible to determine whether the preservation of the gastric mill rhythm generator during different versions of the gastric mill motor pattern that occurs in the isolated STNS accurately reflects the comparable situation in the behaving animal.

\section{References}

Ayali A, Harris-Warrick RM (1999) Monoamine control of the pacemaker kernel and cycle frequency in the lobster pyloric network. J Neurosci 19:6712-6722.

Bartos M, Nusbaum MP (1997) Intercircuit control of motor pattern modulation by presynaptic inhibition. J Neurosci 17:2247-2256.

Bartos M, Manor Y, Nadim F, Marder E, Nusbaum MP (1999) Coordination of fast and slow rhythmic neuronal circuits. J Neurosci 19:66506660 .

Beenhakker MP, Nusbaum MP (2004) Mechanosensory activation of a motor circuit by coactivation of two projection neurons. J Neurosci 24: 6741-6750.

Beenhakker MP, Blitz DM, Nusbaum MP (2004) Long-lasting activation of rhythmic neuronal activity by a novel mechanosensory system in the crustacean stomatogastric nervous system. J Neurophysiol 91:78-91.

Berkowitz A, Roberts A, Soffe SR (2010) Roles for multifunctional and specialized spinal interneurons during motor pattern generation in tadpoles, zebrafish larvae, and turtles. Front Behav Neurosci 4:36.

Bernasconi P, Kohl J (1993) Analysis of co-ordination between breathing and exercise rhythms in man. J Physiol 471:693-706.

Blitz DM, Nusbaum MP (2008) State-dependent presynaptic inhibition regulates central pattern generator feedback to descending inputs. J Neurosci 28:9564-9574.

Blitz DM, White RS, Saideman SR, Cook A, Christie AE, Nadim F, Nusbaum MP (2008) A newly identified extrinsic input triggers a distinct gastric mill rhythm via activation of modulatory projection neurons. J Exp Biol 211:1000-1011

Briggman KL, Kristan WB (2008) Multifunctional pattern-generating circuits. Annu Rev Neurosci 31:271-294.

Bucher D, Taylor AL, Marder E (2006) Central pattern generating neurons simultaneously express fast and slow rhythmic activities in the stomatogastric ganglion. J Neurophysiol 95:3617-3632.

Büschges A, Akay T, Gabriel JP, Schmidt J (2008) Organizing network action for locomotion: insights from studying insect walking. Brain Res Rev 57:162-171.

Cangiano L, Grillner S (2003) Fast and slow locomotor burst generation in the hemispinal cord of the lamprey. J Neurophysiol 89:2931-2942.

Cangiano L, Grillner S (2005) Mechanisms of rhythm generation in a spinal locomotor network deprived of crossed connections: the lamprey hemicord. J Neurosci 25:923-935.

Clemens S, Combes D, Meyrand P, Simmers J (1998) Long-term expression of two interacting motor pattern-generating networks in the stomatogastric system of freely behaving lobster. J Neurophysiol 79:1396-1408.

Coleman MJ, Nusbaum MP (1994) Functional consequences of compartmentalization of synaptic input. J Neurosci 14:6544-6552.

Coleman MJ, Meyrand P, Nusbaum MP (1995) Presynaptic inhibition mediates a switch between two modes of synaptic transmission. Nature 378:502-505.

Combes D, Meyrand P, Simmers J (1999) Motor pattern specification by dual descending pathways to a lobster rhythm-generating network. J Neurosci 19:3610-3619.

Doi A, Ramirez JM (2008) Neuromodulation and the orchestration of the respiratory rhythm. Respir Physiol Neurobiol 164:96-104.

Ezure K, Tanaka I (1997) Convergence of central respiratory and locomotor rhythms onto single neurons of the lateral reticular nucleus. Exp Brain Res 113:230-242.

Friedman AK, Zhurov Y, Ludwar BCh, Weiss KR (2009) Motor outputs in a multitasking network: relative contributions of inputs and experiencedependent network states. J Neurophysiol 102:3711-3727.

Gariépy JF, Missaghi K, Dubuc R (2010) The interactions between locomotion and respiration. Prog Brain Res 187:173-188.

Getting PA, Dekin MS (1985) Mechanisms of pattern generation underlying swimming in Tritonia. IV. Gating of central pattern generator. J Neurophysiol 53:466-480.

Hedrich UB, Diehl F, Stein W (2011) Gastric and pyloric motor pattern control by a modulatory projection neuron in the intact crab, Cancer pagurus. J Neurophysiol 105:1671-1680.

Heinzel HG, Weimann JM, Marder E (1993) The behavioral repertoire of 
the gastric mill in the crab, Cancer pagurus: an in situ endoscopic and electrophysiological examination. J Neurosci 13:1793-1803.

Hooper SL, Marder E (1987) Modulation of the lobster pyloric rhythm by the peptide proctolin. J Neurosci 7:2097-2112.

Huguenard JR, McCormick DA (2007) Thalamic synchrony and dynamic regulation of global forebrain oscillations. Trends Neurosci 30:350-356.

Jing J, Weiss KR (2002) Interneuronal basis of the generation of related but distinct motor programs in Aplysia: implications for current neuronal models of vertebrate intralimb coordination. J Neurosci 22:6228-6238.

Katz PS, Sakurai A, Clemens S, Davis D (2004) Cycle period of a network oscillator is independent of membrane potential and spiking activity in individual central pattern generator neurons. J Neurophysiol 92:19041917.

Kawahara K, Kumagai S, Nakazono Y, Miyamoto Y (1989) Coupling between respiratory and stepping rhythms during locomotion in decerebrate cats. J Appl Physiol 67:110-115.

Kilman VL, Marder E (1996) Ultrastructure of the stomatogastric ganglion neuropil of the crab, Cancer borealis. J Comp Neurol 374:362-375.

Kirby MS, Nusbaum MP (2007) Central nervous system projections to and from the commissural ganglion of the crab Cancer borealis. Cell Tissue Res 328:625-637.

Klein DA, Patino A, Tresch MC (2010) Flexibility of motor pattern generation across stimulation conditions by the neonatal rat spinal cord. J Neurophysiol 103:1580-1590.

Li WC, Roberts A, Soffe SR (2010) Specific brainstem neurons switch each other into pacemaker mode to drive movement by activating NMDA receptors. J Neurosci 30:16609-16620.

Mann EO, Paulsen O (2007) Role of GABAergic inhibition in hippocampal network oscillations. Trends Neurosci 30:343-349.

Marder E, Bucher D (2001) Central pattern generators and the control of rhythmic movements. Curr Biol 11:R986-R996.

Marder E, Bucher D (2007) Understanding circuit dynamics using the stomatogastric nervous system of lobsters and crabs. Annu Rev Physiol 69:291-316.

Marder E, Calabrese RL (1996) Principles of rhythmic motor pattern generation. Physiol Rev 76:687-717.

Marder E, Bucher D, Schulz DJ, Taylor AL (2005) Invertebrate central pattern generation moves along. Curr Biol 15:R685-R699.

Masino MA, Calabrese RL (2002) A functional asymmetry in the leech heartbeat timing network is revealed by driving the network across various cycle periods. J Neurosci 22:4418-4427.

Morin D, Viala D (2002) Coordinations of locomotor and respiratory rhythms in vitro are critically dependent on hindlimb sensory inputs. J Neurosci 22:4756-4765.

Nadim F, Manor Y, Nusbaum MP, Marder E (1998) Frequency regulation of a slow rhythm by a fast periodic input. J Neurosci 18:5053-5067.

Norris BJ, Coleman MJ, Nusbaum MP (1994) Recruitment of a projection neuron determines gastric mill motor pattern selection in the stomatogastric nervous system of the crab, Cancer borealis. J Neurophysiol 72:1451-1463.

Nusbaum MP, Beenhakker MP (2002) A small systems approach to motor pattern generation. Nature 417:343-350.

Peña F, Parkis MA, Tryba AK, Ramirez JM (2004) Differential contribution of pacemaker properties to the generation of respiratory rhythms during normoxia and hypoxia. Neuron 43:105-117.

Pirtle TJ, Satterlie RA (2006) The contribution of the pleural type 12 in- terneuron to swim acceleration in Clione limacina. Invert Neurosci 6:161-168.

Popescu IR, Frost WN (2002) Highly dissimilar behaviors mediated by a multifunctional network in the marine mollusk Tritonia diomedea. J Neurosci 22:1985-1993.

Proekt A, Jing J, Weiss KR (2007) Multiple contributions of an inputrepresenting neuron to the dynamics of the Aplysia feeding network. J Neurophysiol 97:3046-3056.

Rauscent A, Einum J, Le Ray D, Simmers J, Combes D (2009) Opposing aminergic modulation of distinct spinal locomotor circuits and their functional coupling during amphibian metamorphosis. J Neurosci 29:1163-1174.

Rosenbaum P, Wosnitza A, Büschges A, Gruhn M (2010) Activity patterns and timing of muscle activity in the forward walking and backward walking stick insect Carausius morosus. J Neurophysiol 104:1681-1695.

Saideman SR, Blitz DM, Nusbaum MP (2007) Convergent motor patterns from divergent circuits. J Neurosci 27:6664-6674.

Sasaki K, Brezina V, Weiss KR, Jing J (2009) Distinct inhibitory neurons exert temporally specific control over activity of a motoneuron receiving concurrent excitation and inhibition. J Neurosci 29:11732-11744.

Saunders SW, Rath D, Hodges PW (2004) Postural and respiratory activation of the trunk muscles changes with mode and speed of locomotion. Gait Posture 20:280-290.

Selverston AI (2010) Invertebrate central pattern generator circuits. Philos Trans R Soc Lond B Biol Sci 365:2329-2345.

Selverston AI, Miller JP (1980) Mechanisms underlying pattern generation in lobster stomatogastric ganglion as determined by selective inactivation of identified neurons. I. Pyloric system. J Neurophysiol 44:1102-1121.

Stein W (2009) Modulation of stomatogastric rhythms. J Comp Physiol A Neuroethol Sens Neural Behav Physiol 195:989-1009.

Stein W, Smarandache CR, Nickmann M, Hedrich UB (2006) Functional consequences of activity-dependent synaptic enhancement at a crustacean neuromuscular junction. J Exp Biol 209:1285-1300.

Steriade M (2006) Grouping of brain rhythms in corticothalamic systems. Neuroscience 137:1087-1106.

Syed NI, Winlow W (1991) Coordination of locomotor and cardiorespiratory networks of Lymnaea stagnalis by a pair of identified interneurones. J Exp Biol 158:37-62.

Thuma JB, Hooper SL (2002) Quantification of gastric mill network effects on a movement related parameter of pyloric network output in the lobster. J Neurophysiol 87:2372-2384.

Weaver AL, Roffman RC, Norris BJ, Calabrese RL (2010) A role for compromise: synaptic inhibition and electrical coupling interact to control phasing in the leech heartbeat CPG. Front Behav Neurosci 4.pii:38.

Weimann JM, Marder E (1994) Switching neurons are integral members of multiple oscillatory networks. Curr Biol 4:896-902.

Weimann JM, Meyrand P, Marder E (1991) Neurons that form multiple pattern generators: identification and multiple activity patterns of gastric/ pyloric neurons in the crab stomatogastric system. J Neurophysiol 65:111-122.

Welsh DK, Takahashi JS, Kay SA (2010) Suprachiasmatic nucleus: cellautonomy and network properties. Annu Rev Physiol 72:551-577.

White RS, Blitz DM, Nusbaum MP (2007) Different outputs from the same CPG elicit distinct muscle activity patterns. Soc Neurosci Abstr 33:924.22.

Wood DE, Manor Y, Nadim F, Nusbaum MP (2004) Intercircuit control via rhythmic regulation of projection neuron activity. J Neurosci 24:74557463. 